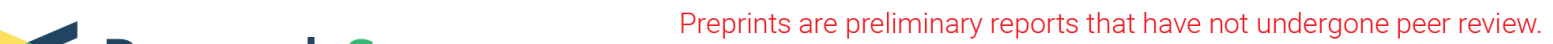 Research Square
or referenced by the media as validated information.
}

\section{Correlation of the Lipid Ratios With Hepatic Steatosis and Liver Fibrosis in Non-alcoholic Fatty Liver Disease Patients}

\section{Wen Guo}

Jiangsu Province People's Hospital and Nanjing Medical University First Affiliated Hospital https://orcid.org/0000-0002-6456-6113

Pei Qin Jiangsu Province People's Hospital and Nanjing Medical University First Affiliated Hospital

Xiaona Li

Jiangsu Province People's Hospital and Nanjing Medical University First Affiliated Hospital

Jing Lu

Jiangsu Province People's Hospital and Nanjing Medical University First Affiliated Hospital

Wenfang Zhu

Jiangsu Province People's Hospital and Nanjing Medical University First Affiliated Hospital

Juan $\mathrm{Wu}$

Jiangsu Province People's Hospital and Nanjing Medical University First Affiliated Hospital

Nainzhen Xu

Jiangsu Province People's Hospital and Nanjing Medical University First Affiliated Hospital

Qun Zhang ( $\square$ wenzi20100305@126.com )

Department of Health Promotion Center The First Affiliated Hospital with Nanjing Medical University 300 Guangzhou Road, Nanjing, China, 210029. https://orcid.org/0000-0003-2208-7998

\section{Research}

Keywords: Lipid ratios, non-alcoholic fatty liver disease, the severity of hepatic steatosis, liver fibrosis

Posted Date: November 19th, 2020

DOI: https://doi.org/10.21203/rs.3.rs-109731/v1

License: (c) (1) This work is licensed under a Creative Commons Attribution 4.0 International License.

Read Full License 


\section{Abstract}

Background: The work intends to investigate the correlation of the lipid ratios to the severity of hepatic steatosis and the presence of liver fibrosis among non-alcoholic fatty liver disease (NAFLD) patients.

Methods: Randomly selected 3402 participants were differentiated into the NAFLD $(n=2036)$ and the non-NAFLD group $(n=1366)$ in accordance with the outcomes of the liver ultrasonography. The related anthropometric and biochemical parameters were measured, while the severity of hepatic steatosis and the presence of liver stiffness were appraised by transient elastography.

Results: The triglycerides/ high-density lipoprotein cholesterol (TG/HDL-C) and total cholesterol/HDL-C (TC/HDL-C) ratios exhibited a close connection with the severity of hepatic steatosis. Furthermore, higher area under the receiver operator characteristic (AUROC) value for TG/HDL-C than those of TC, TG, LDL-C, HDL-C, TC/HDL-C and non-HDL-C, was observed. Accordingly, the AUROC and optimal cut-off point of TG/HDL-C for NAFLD were 0.771 (95\%Cl: 0.755-0.787), 1.08 (sensitivity: $72.2 \%$, specificity: $68.3 \%$ ), respectively. TG/HDL-C and TC/HDL-C were independent risk factors for the presence of liver fibrosis in NAFLD. Notably, the fact of relatively high AUROC while low AUROC (0.610) values of TG/HDL-C for detecting the presence of liver fibrosis relative to other lipid indexes indicated that it could not function as a desirable indicator of the presence of liver fibrosis in NAFLD.

Conclusions: TG/HDL-C can be an independent risk factor for the severity of hepatic steatosis and the presence of liver fibrosis in NAFLD, furthermore, it possesses predictive significance to NAFLD but not to liver fibrosis.

\section{Background}

As a principal chronic disorder for liver, non-alcoholic fatty liver disease (NAFLD) consists of a series of clinical conditions covering simple liver steatosis to non-alcoholic steatohepatitis (NASH), liver fibrosis, cirrhosis and hepatocellular carcinoma. With the improvement of people's living standard and rapid lifestyle transitions, China experiences an unforeseen prompt increase in the burden of NAFLD within a short time and the prevalence of NAFLD reaches to $29.2 \%$ in China [1]. Furthermore, China will appear the fastest growth in the prevalence of NAFLD, with cases of 314.58 million up to 2030 [2], representing a substantial clinical burden and a public health concern. Besides the key cause for liver-related morbidity and mortality, accumulating evidence has shown that NAFLD was closely associated with metabolic syndrome, type 2 diabetes (T2DM) and cardiovascular disease (CVD) [3-5]. Thus, identifying individuals who face a risk to potentially develop to NAFLD is of significance to solve this public health problem.

NAFLD is often related to and accompanied by lipid abnormalities, including high-density lipoprotein cholesterol (HDL-C), triglycerides(TG) and non-HDL-C[6]. During recent years, the concept of the lipid ratios, i.e. total cholesterol (TC)/HDL-C, TG/HDL-C, have been proposed as alternative biomarkers for predicting insulin resistance (IR) $[7,8]$, which plays a crucial role in the pathogenesis of NAFLD. In light of this theory, the relations between TC/HDL-C, TG/HDL-C and NAFLD have extensively been characterized. 
For instance, a cross-sectional, retrospective study revealed that adults with individually or combinedly elevated TC/HDL-C or TG/HDL-C ratios, possessed superior potential for the development of NAFLD, in particular under advanced status [9]. Similarly, another report based on a Chinese community population demonstrated that the independent relation of TG/HDL-C with NAFLD in apparently healthy individuals and may be used as a surrogate for NAFLD [10]. In spite of being the gold criterion to diagnose NASH and its associated progressive fibrosis, the clinical application of liver biopsy is limited due to its invasion and high cost. As a non-invasive approach, Transient elastography (TE) was widely utilized for the prognosis steatosis grades in NAFLD patients in accordance with the Ultrasound Attenuated Parameter (UAP) in several studies. At the same time, liver stiffness measurement (LSM) has been used to decide the developmental stages of fibrosis [11]. Despite these, the data characterizing the connection of the lipid ratios with the severity of hepatic steatosis and the presence of liver fibrosis in NAFLD are limited

The aim of this study intends to explore the issue described above. Additionally, the predictive implication of the lipid ratios and those long-time used lipid indicators of TC, low-density lipoprotein cholesterol (LDLC), TG and HDL-C, are compared and evaluated.

\section{Materials And Methods}

\section{Study population}

A total of 3402 participants ever enrolled in a health examination between May 2017 and July 2019 were randomly selected from the Health Promotion Center of the First Affiliated Hospital of Nanjing Medical University. All participants were further divided into the NAFLD $(n=2036)$ and the non-NAFLD group $(n=$ 1366) in accordance with the outcomes of liver ultrasonography. Exclusion criteria for all participants were: 1) subjects consumed significant alcohol (> $140 \mathrm{~g} /$ week and > $70 \mathrm{~g} /$ week for men and women, respectively); 2) subjects suffered from hepatitis $B$ or $C$ virus infection or from pre-existing chronic liver disease (eg, autoimmune liver disease, primary sclerosing cholangitis, drug-induced liver disease);3) subjects taking lipid-lowering drugs such as statin; 4) hyperthyroidism, kidney disorders, and other lipid metabolism dysfunction associated diseases. The study was approved by the Human Research Ethics Committee of the First Affiliated Hospital of Nanjing Medical University. The informed consent requirement was exempted because of the retrospective study.

\section{Physical Examination And Biochemical Tests}

Weight, height, waist circumference and blood pressure were measured in all participants. Following overnight fasting, collected venous blood specimens were examined for the serum lipids, glucose, hepatic function and uric acid were measured by enzymatic methods (Chemistry Analyzer Au2700, Olympus Medical Engineering Company, Japan). Glycated hemoglobin A1c ( $\mathrm{HbA1c}$ ) values were defined by highperformance liquid chromatography. Liver ultrasonography was performed in all participants. Non-HDL-C 
is equal to TC minus $\mathrm{HDL}-\mathrm{C}$, while the $\mathrm{TC} / \mathrm{HDL}-\mathrm{C}$ or $\mathrm{TG} / \mathrm{HDL}-\mathrm{C}$ ratio is equal to $\mathrm{TC}$ or $\mathrm{TG}$ dividing $\mathrm{HDL}-\mathrm{C}$, respectively.

\section{Liver Stiffness And Steatosis}

Transient elastography was carried out using the FibroTouch (Wuxi Hisky Medical Technologies Co., Ltd., China) according to the operations manual. The extent of liver fibrosis and hepatic steatosis was determined by liver stiffness measurement (LSM) and fat attenuation parameter (FAP). According to the FAP value, NAFLD patients were subdivided into three groups: the mild $(240 \mathrm{~dB} / \mathrm{m} \leq \mathrm{FAP}<265 \mathrm{~dB} / \mathrm{m})$, the moderate $(265 \mathrm{~dB} / \mathrm{m} \leq \mathrm{FAP}<295 \mathrm{~dB} / \mathrm{m})$ and the severe NAFLD group (FAP $\geq 295 \mathrm{~dB} / \mathrm{m})$. According to the LSM value, patients with NAFLD were also subdivided into two groups: the non-liver ( $L S M \leq 7.3 \mathrm{Kpa})$ and the liver fibrosis group (LSM > 7.3 Kpa).

\section{Statistical analysis}

Mean \pm SD represented continuous variables. T-test was applied to examine differences between two groups, while one-way ANOVA with Bonferroni correction for pairwise comparisons was utilized to examine differences among the three groups. Pearson's qui-squared test was applied to assess categorical variables presented as percentages (numbers). The binary or multinomial logistic regression analysis was carried out to explore the independent indicators for NAFLD and liver fibrosis. Receiver operator characteristic (ROC) analyses were performed to calculate area under the ROC curve (AUROC) of each lipid parameter for incident NAFLD and liver fibrosis. SPSS18.0 statistical software was used to analyse all data, with $p<0.05$ denoting statistical significance (two-sided).

\section{Results}

\section{Baseline characteristics of the population involved in this study}

Clinical and biochemical characteristics of the participants were listed in Table 1, NAFLD patients tended to be men and possessed higher body mass index (BMI), systolic blood pressure (SBP), diastolic blood pressure (DBP), fasting blood glucose (FBG), HbA1c, TC, TG, LDL-C, TC/HDL-C, TG/HDL-C, non-HDL-C, serum uric acid, ALT, AST, GGT while lower HDL-C (all $P<0.05)$ when compared to non-NAFLD individuals. Notably, no difference existed in terms of age between the two groups. 
Table 1

Baseline characteristics of individuals with or without NAFLD

\begin{tabular}{|c|c|c|}
\hline & Non-NAFLD $(n=1366)$ & NAFLD $(n=2036)$ \\
\hline Age(years) & $49.02 \pm 10.81$ & $49.42 \pm 10.08$ \\
\hline Sex(male/Female) & $689 / 677$ & $1577 / 459^{\star}$ \\
\hline BMI (kg/m2) & $22.48 \pm 3.77$ & $26.61 \pm 4.55^{*}$ \\
\hline $\mathrm{SBP}(\mathrm{mmHg})$ & $122.85 \pm 17.69$ & $130.74 \pm 16.43$ * \\
\hline $\mathrm{DBP}(\mathrm{mmHg})$ & $74.67 \pm 11.15$ & $81.17 \pm 10.95^{*}$ \\
\hline FBG (mmol/L) & $5.26 \pm 1.06$ & $5.77 \pm 1.39^{*}$ \\
\hline HbA1c (\%) & $5.53 \pm 0.62$ & $5.80 \pm 0.87^{\star}$ \\
\hline $\mathrm{TC}(\mathrm{mmol} / \mathrm{L})$ & $5.28 \pm 1.04$ & $5.41 \pm 1.06$ * \\
\hline $\mathrm{TG}(\mathrm{mmol} / \mathrm{L})$ & $1.30 \pm 0.59$ & $2.02 \pm 1.10^{\star}$ \\
\hline LDL-C(mmol/L) & $3.23 \pm 0.77$ & $3.44 \pm 0.76^{*}$ \\
\hline $\mathrm{HDL}-\mathrm{C}(\mathrm{mmol} / \mathrm{L})$ & $1.44 \pm 0.32$ & $1.22 \pm 0.25^{\star}$ \\
\hline TG/HDL-C & $0.98 \pm 0.56$ & $1.77 \pm 1.17^{\star}$ \\
\hline TC/HDL-C & $3.78 \pm 0.89$ & $4.54 \pm 0.97^{\star}$ \\
\hline non-HDL-C & $3.84 \pm 0.97$ & $4.19 \pm 0.98^{\star}$ \\
\hline Uric acid (mmol/l) & $310.80 \pm 80.71$ & $370.73 \pm 84.23^{*}$ \\
\hline $\mathrm{ALT}(\mathrm{U} / \mathrm{L})$ & $19.85 \pm 10.71$ & $29.97 \pm 17.09^{\star}$ \\
\hline AST (U/L) & $22.46 \pm 7.48$ & $24.87 \pm 8.44^{*}$ \\
\hline GGT (U/L) & $25.98 \pm 19.87$ & $41.96 \pm 28.07^{\star}$ \\
\hline \multicolumn{3}{|c|}{ Values are presented as mean \pm standard deviation. } \\
\hline \multicolumn{3}{|c|}{$\begin{array}{l}\text { BMI, body mass index; SBP, systolic blood pressure; DBP, diastolic blood pressure; FBG, fasting blood } \\
\text { glucose; TC, total cholesterol; TG, triacylglyceride; LDL-C, high-density lipoprotein cholesterol; HDL-C, } \\
\text { high-density lipoprotein cholesterol; ALT: alanine aminotransferase; AST aspartate transaminase; } \\
\text { GGT: gamma-glutamyl transpeptidase. Compared with non-NAFLD, }{ }^{\star} P<0.05\end{array}$} \\
\hline
\end{tabular}

\section{Lipid Ratios Are Associated With Hepatic Steatosis}


In accordance with the FAP value, NAFLD patients were further divided into three groups: the mild $(\mathrm{n}=$ $735)$, the moderate $(n=559)$ and the severe NAFLD group $(n=742)$. Interestingly, there were pronouncedly differences in TG, TC/HDL-C, TG/HDL-C and HDL-C among the mild, moderate, and severe NAFLD groups $(P<0.05)$ (Fig. 1). Furthermore, patients in the moderate/severe group exhibited higher non-HDL-C than that in the mild NAFLD group $(P<0.05)$ (Fig. 1), whereas patients of the severe group displayed elevated TC relative to those in the mild/moderate NAFLD group $(P<0.05)($ Fig. 1$)$.

\section{Baseline characteristics of individuals with or without liver fibrosis in NAFLD}

In line with the LSM value, NAFLD patients were further differentiated into the non-liver $(n=1665)$ and liver fibrosis groups $(n=371)$. As listed in Table 2, patients with liver fibrosis similarly tended to be men and displayed higher parameters in BMI, SBP, DBP, FBG, HbA1c, TG, TC/HDL-C, TG/HDL-C, non-HDL-C, serum uric acid, ALT, AST, GGT while lower HDL-C (all $P<0.05)$ relative to individuals without liver fibrosis. Likewise, these two groups exhibited no differences of age, TC and LDL-C. 
Table 2

Baseline characteristics of individuals with or without liver fibrosis in NAFLD

\begin{tabular}{|c|c|c|}
\hline & Non-liver fibrosis $(n=1665)$ & liver fibrosis( $n=371)$ \\
\hline Age(years) & $49.29 \pm 9.88$ & $50.02 \pm 10.91$ \\
\hline Sex(male/Female) & $1272 / 293$ & $305 / 66^{*}$ \\
\hline $\mathrm{BMI}(\mathrm{kg} / \mathrm{m} 2)$ & $26.14 \pm 4.43$ & $28.70 \pm 4.47^{\star}$ \\
\hline $\mathrm{SBP}(\mathrm{mmHg})$ & $129.66 \pm 16.16$ & $135.43 \pm 16.80$ * \\
\hline $\mathrm{DBP}(\mathrm{mmHg})$ & $80.68 \pm 10.95$ & $83.34 \pm 10.73^{*}$ \\
\hline FBG $(\mathrm{mmol} / \mathrm{L})$ & $5.67 \pm 1.30$ & $6.18 \pm 1.68^{\star}$ \\
\hline HbA1c (\%) & $5.74 \pm 0.81$ & $6.06 \pm 1.09^{\star}$ \\
\hline $\mathrm{TC}(\mathrm{mmol} / \mathrm{L})$ & $5.40 \pm 1.06$ & $5.48 \pm 1.07$ \\
\hline TG (mmol/L) & $1.93 \pm 0.90$ & $2.44 \pm 1.68^{*}$ \\
\hline LDL-C(mmol/L) & $3.43 \pm 0.77$ & $3.47 \pm 0.75$ \\
\hline $\mathrm{HDL}-\mathrm{C}(\mathrm{mmol} / \mathrm{L})$ & $1.23 \pm 0.24$ & $1.17 \pm 0.25^{\star}$ \\
\hline TG/HDL-C & $1.66 \pm 0.91$ & $2.23 \pm 1.88^{\star}$ \\
\hline TC/HDL-C & $4.47 \pm 0.94$ & $4.83 \pm 1.09^{\star}$ \\
\hline non-HDL-C & $4.16 \pm 0.98$ & $4.32 \pm 0.99^{\star}$ \\
\hline Uric acid (mmol/l) & $367.33 \pm 83.91$ & $385.98 \pm 84.02^{*}$ \\
\hline $\mathrm{ALT}(\mathrm{U} / \mathrm{L})$ & $28.42 \pm 15.92$ & $36.97 \pm 20.17^{\star}$ \\
\hline AST (U/L) & $24.12 \pm 7.81$ & $28.22 \pm 10.19^{*}$ \\
\hline GGT (U/L) & $40.56 \pm 27.31$ & $48.28 \pm 30.48^{*}$ \\
\hline \multicolumn{3}{|c|}{ Values are presented as mean \pm standard deviation. } \\
\hline \multicolumn{3}{|c|}{$\begin{array}{l}\text { BMI, body mass index; SBP, systolic blood pressure; DBP, diastolic blood pressure; FBG, fasting blood } \\
\text { glucose; TC, total cholesterol; TG, triacylglyceride; LDL-C, high-density lipoprotein cholesterol; HDL-C, } \\
\text { high-density lipoprotein cholesterol; ALT: alanine aminotransferase; AST aspartate transaminase; } \\
\text { GGT: gamma-glutamyl transpeptidase. Compared with non-liver fibrosis, }{ }^{\star} P<0.05\end{array}$} \\
\hline
\end{tabular}

Multinomial logistic regression assessment the risk of hepatic steatosis severity 
After adjusting for sex, BMI, SBP, DBP, FBG, HbA1c, uric acid, ALT, AST and GGT, TG, HDL-C, TG/HDL-C, TC/HDL-C and non-HDL-C were all significantly correlated with the severity of hepatic steatosis. Briefly, compared to those without NAFLD, the odds ratios of TG for the mild, moderate and severe NAFLD were 1.904 (95\% Cl1.610-2.251, $P<0.001$ ), 2.173 (95\% C/1.817-2.600, $P<0.001$ ), 2.512 (95\% Cl2.090-3.019, $P<0.001)$, respectively. The odds ratios of HDL-C for the mild, moderate and severe NAFLD were 0.355 (95\% Cl 0.232-0.543, $P<0.001$ ), 0.260 (95\%Cl 0.156-0.432, $P<0.001$ ), 0.178 (95\%Cl 0.101-0.313, $P<$ 0.001 ), respectively. The odds ratios of TG/HDL-C for mild, moderate and severe NAFLD were 1.967 (95\%Cl1.659-2.333, $P<0.001), 2.314$ (95\%Cl1.930-2.773, $P<0.001), 2.570$ (95\%Cl2.134-3.095, $P<$ 0.001), respectively. The odds ratios of TC/HDL-C for mild, moderate and severe NAFLD were 1.301 (95\%Cl1.149-1.472, $P<0.001), 1.609$ (95\% Cl1.398-1.851, $P<0.001)$ and $1.775(95 \% C / 1.526-2.065, P$ $<0.001$ ), respectively. The odds ratios of non-HDL-C for mild, moderate and severe NAFLD were 1.093 (95\% Cl 0.978-1.222, $P=0.116), 1.277$ (95\% Cl 1.125-1.450, $P<0.001), 1.341$ (95\%Cl1.169-1.538, $P<$ $0.001)$, respectively.

\section{Binary logistic regression analysis for the risk of liver fibrosis in NAFLD}

In line with binary logistic regression analysis, TG $(O R=1.341,95 \% C / 1.195-1.505, P<0.001), \mathrm{HDL}-\mathrm{C}(O R$ $=0.512,95 \% C / 0.292-0.895, P=0.019), \mathrm{TG} / \mathrm{HDL}-\mathrm{C}(O R=1.322,95 \% C / 1.178-1.483, P<0.001)$ and $\mathrm{TC} / \mathrm{HDL}-\mathrm{C}(O R=1.252,95 \% \mathrm{Cl} 1.098-1.427, P=0.001)$ presented a positive correlation with liver fibrosis upon adjusting for sex, BMI, SBP, DBP, FBG, HbA1c, uric acid, ALT, AST and GGT. On the contrary, there were no obvious relationship of NAFLD with TC, LDL-C, or non-HDL-C following the adjustment for sex, BMI, SBP, DBP, FBG, HbA1c, uric acid, ALT, AST and GGT.

\section{Diagnostic Value Of Lipid Parameters For Nafld}

The cut-off points of lipid parameters for predicting NAFLD, together with their corresponding specificity and sensitivity, were summarized in Table 3 and Fig. 2. Higher AUROC value of TG/HDL-C than those of TC, TG, LDL-C, HDL-C, TC/HDL-C and non-HDL-C was observed. Accordingly, the AUROC and optimal cutoff point of TG/HDL-C was 0.771 (95\% C/ $0.755-0.787$ ) and 1.08 (sensitivity: $72.2 \%$, specificity: $68.3 \%$ ), respectively, suggesting that TG/HDL-C had better predictive effects than other lipid parameters and was an acceptable predictor of NAFLD. 
Table 3

ROC curve for predicting NAFLD and cut-off points for maximum sum of sensitivity and specificity

\begin{tabular}{|llllll|}
\hline & ROC $(95 \%$ Cl & $P$ & Cut-off point & Sensitivity (\%) & Specificity (\%) \\
\hline TC $(\mathrm{mmol} / \mathrm{L})$ & $0.538(0.518-0.558)$ & 0.010 & 5.29 & 0.521 & 0.542 \\
\hline TG $(\mathrm{mmol} / \mathrm{L})$ & $0.748(0.731-0.764)$ & 0.008 & 1.40 & 0.698 & 0.675 \\
\hline LDL-C(mmol/L) & $0.583(0.564-0.603)$ & 0.010 & 3.35 & 0.538 & 0.602 \\
\hline HDL-C(mmol/L) & $0.292(0.274-0.310)$ & 0.009 & 1.30 & 0.641 & 0.676 \\
\hline TG/HDL-C & $0.771(0.755-0.787)$ & 0.008 & 1.08 & 0.722 & 0.683 \\
\hline TC/HDL-C & $0.721(0.703-0.738)$ & 0.009 & 4.03 & 0.681 & 0.649 \\
\hline non-HDL-C & $0.605(0.586-0.625)$ & 0.010 & 3.94 & 0.581 & 0.583 \\
\hline
\end{tabular}

\section{Diagnostic value of lipid parameters for liver fibrosis in NAFLD}

The cut-off points of lipid parameters for predicting liver fibrosis, together with their corresponding specificity and sensitivity, were listed in Table 4 and Fig. 3. AUROC value of TG/HDL-C was larger than that of TG, HDL-C and TC/HDL-C. However, The AUROC value of TG/HDL-C $(0.60<A U R O C<0.70)$ for liver fibrosis was relatively smaller than that for NAFLD, which indicated that TG/HDL-C could not be acceptable predictors of liver fibrosis in NAFLD.

Table 4

ROC curve for predicting liver fibrosis and cut-off points for maximum sum of sensitivity and specificity

\begin{tabular}{|llllll|}
\hline & ROC $(95 \% \mathrm{Cl})$ & $\boldsymbol{P}$ & Cut-off point & Sensitivity (\%) & Specificity (\%) \\
\hline TG (mmol/L) & $0.599(0.567-0.630)$ & 0.016 & 1.97 & 0.515 & 0.607 \\
\hline HDL-C(mmol/L) & $0.415(0.383-0.447)$ & 0.016 & 1.19 & 0.534 & 0.572 \\
\hline TG/HDL-C & $0.610(0.579-0.641)$ & 0.016 & 1.70 & 0.518 & 0.621 \\
\hline TC/HDL-C & $0.597(0.565-0.630)$ & 0.017 & 4.52 & 0.601 & 0.553 \\
\hline
\end{tabular}

\section{Discussion}

We for the first time demonstrated a relationship of the lipid ratios to the severity of NAFLD and the presence of liver fibrosis evaluated by transient elastography in a large cohort study. Importantly, this study provided the major finding of a positive correlation of TG/HDL-C to the severity of hepatic steatosis and the presence of liver fibrosis of NAFLD among the population of apparently healthy individuals. Notably, TG/HDL-C could be a superior biomarker for NAFLD relative to other lipid ratios like TC/HDL-C, while it could not be a promising predictor for liver fibrosis in NAFLD. 
As a burgeoning health problem, NAFLD is currently considered as the commonest prevalent chronic liver problem in China [12]. It has been clear that with the continued growth in the prevalence of obesity and diabetes, increased incidence of a series of liver-related disorders like NASH, cirrhosis, and other liver complications will be witnessed [2]. Moreover, increased NAFLD morbidity might pose great risks to the development of metabolic, cardiovascular and renal diseases $[13,14]$. Therefore, identifying individuals with increased risks developing to NAFLD could solve this important public health issue.

Recently, TG/HDL-C ratio was applied to clinical setting, mainly including predicting T2DM, IR and cardiovascular diseases $[15,16]$, which are all related to NAFLD. The association between TG/HDL-C and NAFLD has gained more interest from many scholars. A retrospective cohort research has indicated that TG/HDL-C had a close relationship with NAFLD within the population of non-obese Chinese individuals lacking of dyslipidemia, and it could be utilized as an effective predictor for NAFLD [17]. Similarly, a cross-sectional study in China has also indicated that TG/HDL-C is independently associated with NAFLD in apparently healthy individuals and can be employed as a surrogate for NAFLD [10]. Consistent with these, the current study also confirmed a significantly positive correlation of TG/HDL-C to NAFLD. Despite these, the investigation on the relationship of the TG/HDL-C with the severity of hepatic steatosis and the presence of liver fibrosis and comparison of the predictive value of TG/ HDL-C with other lipid ratios like TC/HDL-C, has not been performed. This work demonstrated that TG/HDL-C was an independent risk factor for severity of hepatic steatosis and the presence of liver fibrosis in NAFLD even upon adjusting other risk factors such as $\mathrm{BMI}, \mathrm{FBG}$ and $\mathrm{HbA} 1 \mathrm{c}$. Meanwhile, the results from ROC analysis further demonstrated that TG/HDL-C had higher predictive value for NAFLD than other lipid parameters and lipid ratios, implying a potential surrogate for NAFLD. Although the AUROC of TG/HDL-C for detecting liver fibrosis was larger than other lipid indexes, the AUC value of TG/HDL-C was relatively low (AUROC 0.60.7), indicating limited accuracy of TG/HDL-C in predicting liver fibrosis in NAFLD. Though the mechanism behind the connection of TG/HDL-C with NAFLD remains unsolved, we speculated that insulin resistance might be a potential mediator. In clinical studies, the close relation of TG/HDL-C to IR has been identified in various populations including overweight and obese children and adolescents, middle-aged and elderly population, and people with different glucose tolerance status [18-20]. Furthermore, it has been suggested to be a clinically useful tool to assess IR, as confirmed by the fact that IR can boost the secretion of adverse VLDL particles while reduce the concentration of HDL-C in experimental studies $[21,22]$. Thus, IR promotes the elevation of TG/HDL-C, meanwhile, it is fundamentally involved in the NALFD pathogenesis [23]. Therefore, we speculated that IR may establish a connection for TG/HDL-C and NAFLD, whereas the exact molecular mechanisms require further investigations.

Previous study demonstrated that TC/HDL-C can indirectly reflect the number of LDL-C particles and function as a powerful risk indicator for IR, CVD, and T2DM [24]. Furthermore, the Jinchang cohort study showed that TC/HD-C displayed a positive correlation with NAFLD and had significant predictive value for NAFLD than TC, HDL-C and Apolipoprotein B to Apolipoprotein A1 (ApoB/ApoA1) [25]. Higher TC/HDL-C in adults often signified increased risk for NAFLD, thereby implying a role of TC/HDL-C for predicting the incidence of NAFLD [9]. However, these two studies have not addressed the relation of the 
$\mathrm{TC} / \mathrm{HDL}-\mathrm{C}$ to the severity of hepatic steatosis and the presence of liver fibrosis and compared the predictive value of TC/ HDL-C with TG/HDL-C. This work indicated that TC/HDL-C was an independent risk factor for the severity of hepatic steatosis even upon adjusting other risk factors, such as BMI, FBG and $\mathrm{HbA} 1 \mathrm{c}$. ROC analysis results demonstrated that TC/HDL-C (the AUROC $>0.70$ ) was an acceptable predictor for NAFLD, while TC/HDL-C had a lower AUROC than TG/HDL-C. Although TC/HDL-C could be an independent risk factor for liver fibrosis in NAFLD, the AUC value of it was low (AUROC 0.5-0.6), indicating that it could not function as a perfect predictor for liver fibrosis in NAFLD.

Non-HDL-C, consisting of very low-density lipoprotein (VLDL) remnant particles and intermediate density particles, is often used to indirectly estimate the LDL particle number, which is more closely linked with the occurrence of cardiovascular and atherosclerotic events than LDL-C [26]. Zelber-Sagi S et al. found that as an independent predictor for NAFLD, non-HDL-C was superior over TC, TG, HDL-C and LDL-C [27]. Similarly, previous report indicated that pronouncedly increased non-HDL-C were often related to NASH rather than steatosis in patients not receiving lipid-lowering agents [28]. However, the resolution NASH was frequently associated with the improvement in TG and HDL but not non-HDL-C [29]. Our results indicated that even following the adjustment of other risk factors like BMI, FBG and HbA1c, non-HDL-C was remarkedly related to the moderate and severe hepatic steatosis. However, the connections between non-HDL-C and the mild hepatic steatosis or between non-HDL-C and liver fibrosis was absent when adjusting other risk factors. Additional studies with larger populations and gold-standard hepatic biopsy are still needed to verify the relationship of non-HDL-C with NAFLD.

Several limitations exist in this study. Firstly, it is hard to completely define whether the lipid ratios have a causative effect on the severity of hepatic steatosis and liver fibrosis due to the cross-sectional design. Secondly, liver biopsy is considered the gold standard for the determination of steatosis or liver fibrosis, while it is invasive and costly. Additionally, liver biopsy might be impractical in such a large number of subjects who underwent health check-up examinations. By comparison, we used the ultrasonography examination and transient elastography for non-invasive assessment of hepatic steatosis and fibrosis. Thirdly, since serum insulin level was not measured in our study, we cannot explore whether the connection of NAFLD with TG/HDL-C is possibly influenced by IR.

\section{Conclusion}

The evidence provided here show that the lipid ratios, especially TG/HDL-C, are closely linked with increased risk for the severity of hepatic steatosis and the presence of liver fibrosis in NAFLD. This work confirms that TG/HDL-C can be a biomarker for NAFLD, with a greatly promising value. Considering the potential significance of TG/HDL-C to public health, it is important to check peoples whose TG/HDL-C are elevated, especially TG/HDL-C over 1.08 , lifestyles modification is needed for preventing future NAFLD.

\section{Abbreviations}


NAFLD, non-alcoholic fatty liver disease ; NASH, non-alcoholic steatohepatitis; BMI, body mass index; SBP, systolic blood pressure; DBP, diastolic blood pressure; FBG, fasting blood glucose; TC, total cholesterol; TG, triacylglyceride; LDL-C, high-density lipoprotein cholesterol; HDL-C, high-density lipoprotein cholesterol; ALT

alanine aminotransferase; AST:aspartate transaminase; GGT:gamma-glutamyl transpeptidase; LSM, liver stiffness measurement; FAP, fat attenuation parameter; CVD, cardiovascular disease; T2DM, type 2 diabetes; IR, insulin resistance; AUC, Area under the curve; $\mathrm{Cl}$, Confidence intervals

\section{Declarations}

\section{Acknowledgements}

We appreciate the help and support from all participants who took part in the study.

\section{Authors' contributions}

Wen Guo, and Qun Zhang participated in the study design. Wen Guo, Qin Pei, Xiaona Li, Jing Lu, Wenfang Zhu, Juan Wu and Nianzhen Xu were involved in the conduct of the study and data collection. Wen Guo and Qin Pei made contributions to the data analysis and interpretation of the results. Wen Guo and Qun Zhang wrote and modified the manuscript and prepared the tables and figures. All authors read and approved the final manuscript.

\section{Financial support}

Financial support for this project was from National Natural Sciences Foundation of China (No. 81703027), Science and Technology Department of Jiangsu Province (No. BE2016787) and the Jiangsu Provincial Health Planning and Research Project (H2017019).

\section{Availability of data and materials}

Data sharing is not applicable to this article as participants did not consent to this.

\section{Ethics approval and consent to participate}

This study was approved by the Ethics Committee of the First Affiliated Hospital of Nanjing Medical University, and the informed consent requirement was exempted because of the retrospective estimation of de-identified database.

\section{Consent for publication}

Not applicable.

\section{Competing interests}

The authors declare that they have no competing interest.

\section{References}


1. Zhou F, Zhou J, Wang W, et al. Unexpected Rapid Increase in the Burden of NAFLD in China From 2008 to 2018: A Systematic Review and Meta-Analysis. Hepatology 2019; 70(4): 1119-1133.

2. Estes C, Anstee QM, Arias-Loste MT, et al. Modeling NAFLD disease burden in China, France, Germany, Italy, Japan, Spain, United Kingdom, and United States for the period 2016-2030. J Hepatol 2018; 69(4): 896-904.

3. Baratta F, Pastori D, Angelico F, et al. Nonalcoholic Fatty Liver Disease and Fibrosis Associated With Increased Risk of Cardiovascular Events in a Prospective Study. Clin Gastroenterol Hepatol 2019; S1542-3565(19)31506-X.

4. Mantovani A, Byrne CD, Bonora E, Targher G. Nonalcoholic Fatty Liver Disease and Risk of Incident Type 2 Diabetes: A Meta-analysis. Diabetes Care 2018; 41(2): 372-382.

5. Kim D, Touros A, Kim WR. Nonalcoholic Fatty Liver Disease and Metabolic Syndrome. Clin Liver Dis 2018; 22(1): 133-140.

6. Dowla S, Aslibekyan S, Goss A, Fontaine K, Ashraf AP. Dyslipidemia is associated with pediatric nonalcoholic fatty liver disease. J Clin Lipidol 2018; 12(4): 981-987.

7. Zhang L, Chen S, Deng A, et al. Association between lipid ratios and insulin resistance in a Chinese population. PLoS One 2015; 10(1): e0116110.

8. Uruska A, Zozulinska-Ziolkiewicz D, Niedzwiecki P, Pietrzak M, Wierusz-Wysocka B. TG/HDL-C ratio and visceral adiposity index may be useful in assessment of insulin resistance in adults with type 1 diabetes in clinical practice. J Clin Lipidol 2018; 12(3): 734-740.

9. Wu KT, Kuo PL, Su SB, et al. Nonalcoholic fatty liver disease severity is associated with the ratios of total cholesterol and triglycerides to high-density lipoprotein cholesterol. J Clin Lipidol 2016; 10(2): 420-5.e1.

10. Fan N, Peng L, Xia Z, et al. Triglycerides to high-density lipoprotein cholesterol ratio as a surrogate for nonalcoholic fatty liver disease: a cross-sectional study. Lipids Health Dis 2019; 18(1): 39.

11. Sandrin L, Fourquet B, Hasquenoph JM, et al. Transient elastography: a new noninvasive method for assessment of hepatic fibrosis. Ultrasound Med Biol. 2003. 29(12): 1705-13.

12. Wang FS, Fan JG, Zhang Z, Gao B, Wang HY. The global burden of liver disease: the major impact of China. Hepatology 2014; 60(6): 2099-108.

13. Mantovani A, Zaza G, Byrne CD, et al. Nonalcoholic fatty liver disease increases risk of incident chronic kidney disease: A systematic review and meta-analysis. Metabolism 2018; 79: 64-76.

14. Janssen A, Grobbee DE, Dendale P. Non-alcoholic fatty liver disease, a new and growing risk indicator for cardiovascular disease. Eur J Prev Cardiol 2019 : 2047487319891783.

15. Qin H, Chen Z, Zhang Y, et al. Triglyceride to high-density lipoprotein cholesterol ratio is associated with incident diabetes in men: A retrospective study of Chinese individuals. J Diabetes Investig 2020; 11(1): 192-198.

16. Yang M, Rigdon J, Tsai SA. Association of triglyceride to HDL cholesterol ratio with cardiometabolic outcomes. J Investig Med 2019; 67(3): 663-668. 
17. Chen Z, Qin H, Qiu S, Chen G, Chen Y. Correlation of triglyceride to high-density lipoprotein cholesterol ratio with nonalcoholic fatty liver disease among the non-obese Chinese population with normal blood lipid levels: a retrospective cohort research. Lipids Health Dis 2019; 18(1): 162.

18. Locateli JC, Lopes WA, Simões CF, et al. Triglyceride/glucose index is a reliable alternative marker for insulin resistance in South American overweight and obese children and adolescents. J Pediatr Endocrinol Metab 2019; 32(10): 1163-1170.

19. Yeh WC, Tsao YC, Li WC, Tzeng IS, Chen LS, Chen JY. Elevated triglyceride-to-HDL cholesterol ratio is an indicator for insulin resistance in middle-aged and elderly Taiwanese population: a crosssectional study. Lipids Health Dis 2019; 18(1): 176.

20. Zhou M, Zhu L, Cui X, et al. The triglyceride to high-density lipoprotein cholesterol (TG/HDL-C) ratio as a predictor of insulin resistance but not of $\beta$ cell function in a Chinese population with different glucose tolerance status. Lipids Health Dis 2016; 15: 104.

21. Lucero D, Miksztowicz V, Macri V, et al. Overproduction of altered VLDL in an insulin-resistance rat model: Influence of SREBP-1c and PPAR-a. Clin Investig Arterioscler 2015; 27(4): 167-74.

22. Sparks JD, Sparks CE, Adeli K. Selective hepatic insulin resistance, VLDL overproduction, and hypertriglyceridemia. Arterioscler Thromb Vasc Biol 2012; 32(9): 2104-12.

23. Smith GI, Shankaran M, Yoshino M, et al. Insulin resistance drives hepatic de novo lipogenesis in nonalcoholic fatty liver disease. J Clin Invest 2020;130(3):1453-1460.

24. Song Q, Liu X, Wang A, et al. Associations between non-traditional lipid measures and risk for type 2 diabetes mellitus in a Chinese community population: a cross-sectional study. Lipids Health Dis 2016; 15: 70.

25. Ren XY, Shi D, Ding J, et al. Total cholesterol to high-density lipoprotein cholesterol ratio is a significant predictor of nonalcoholic fatty liver: Jinchang cohort study. Lipids Health Dis 2019; 18(1): 47.

26. Carbayo HJA, Simarro RM, Palazón BA, et al. Evaluation of non-HDL cholesterol as a predictor of non-fatal cardiovascular events in a prospective population cohort. Clin Investig Arterioscler 2018; 30(2): 64-71.

27. Zelber-Sagi S, Salomone F, Yeshua H, et al. Non-high-density lipoprotein cholesterol independently predicts new onset of non-alcoholic fatty liver disease. Liver Int 2014; 34(6): e128-35.

28. Corey KE, Lai M, Gelrud LG, et al. Non-high-density lipoprotein cholesterol as a biomarker for nonalcoholic steatohepatitis. Clin Gastroenterol Hepatol 2012; 10(6): 651-6.

29. Corey KE, Vuppalanchi R, Wilson LA, Cummings OW, Chalasani N. NASH resolution is associated with improvements in $\mathrm{HDL}$ and triglyceride levels but not improvement in LDL or non-HDL-C levels. Aliment Pharmacol Ther 2015; 41(3): 301-9.

\section{Figures}



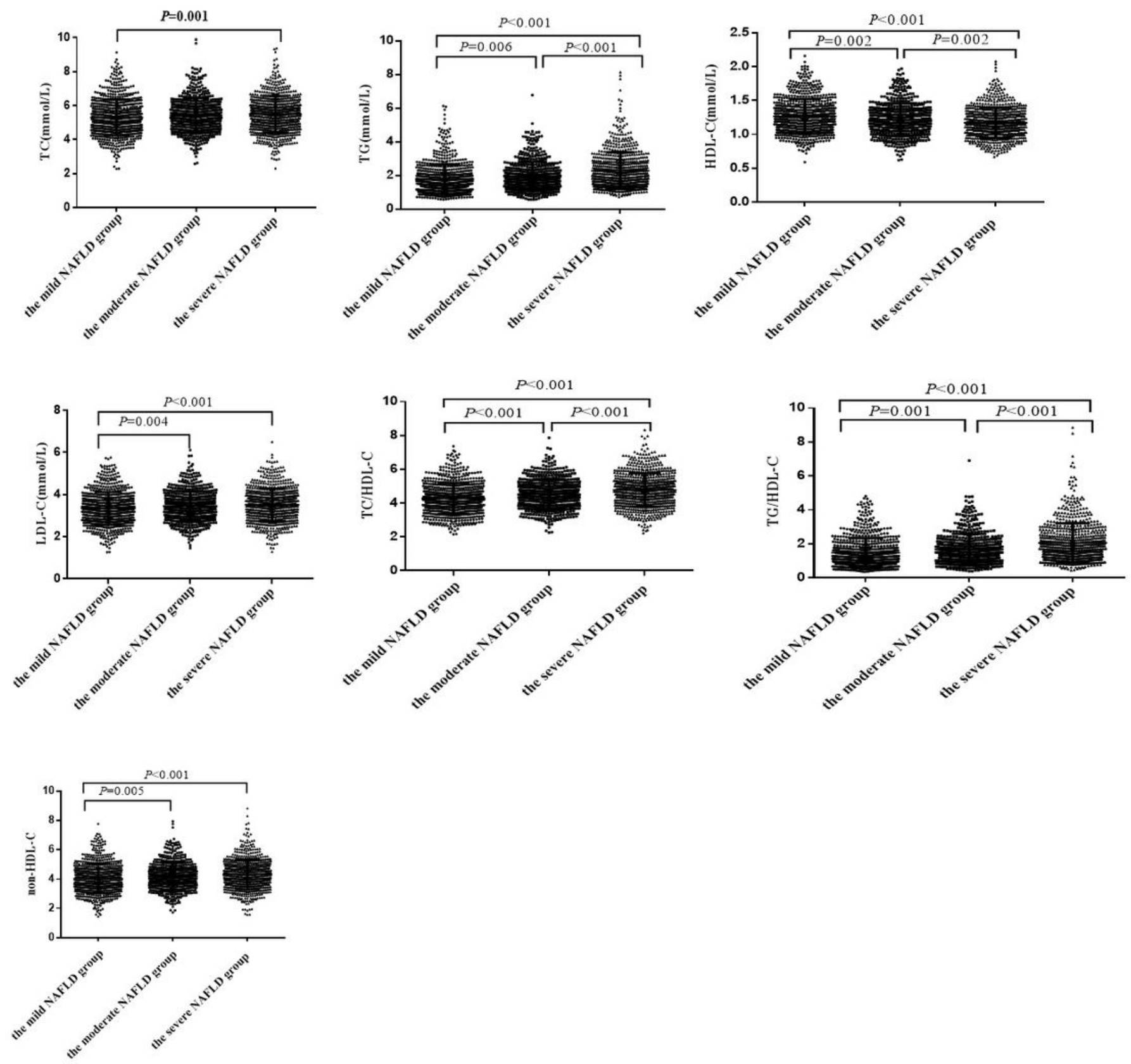

\section{Figure 1}

Lipid ratios are associated with hepatic steatosis 


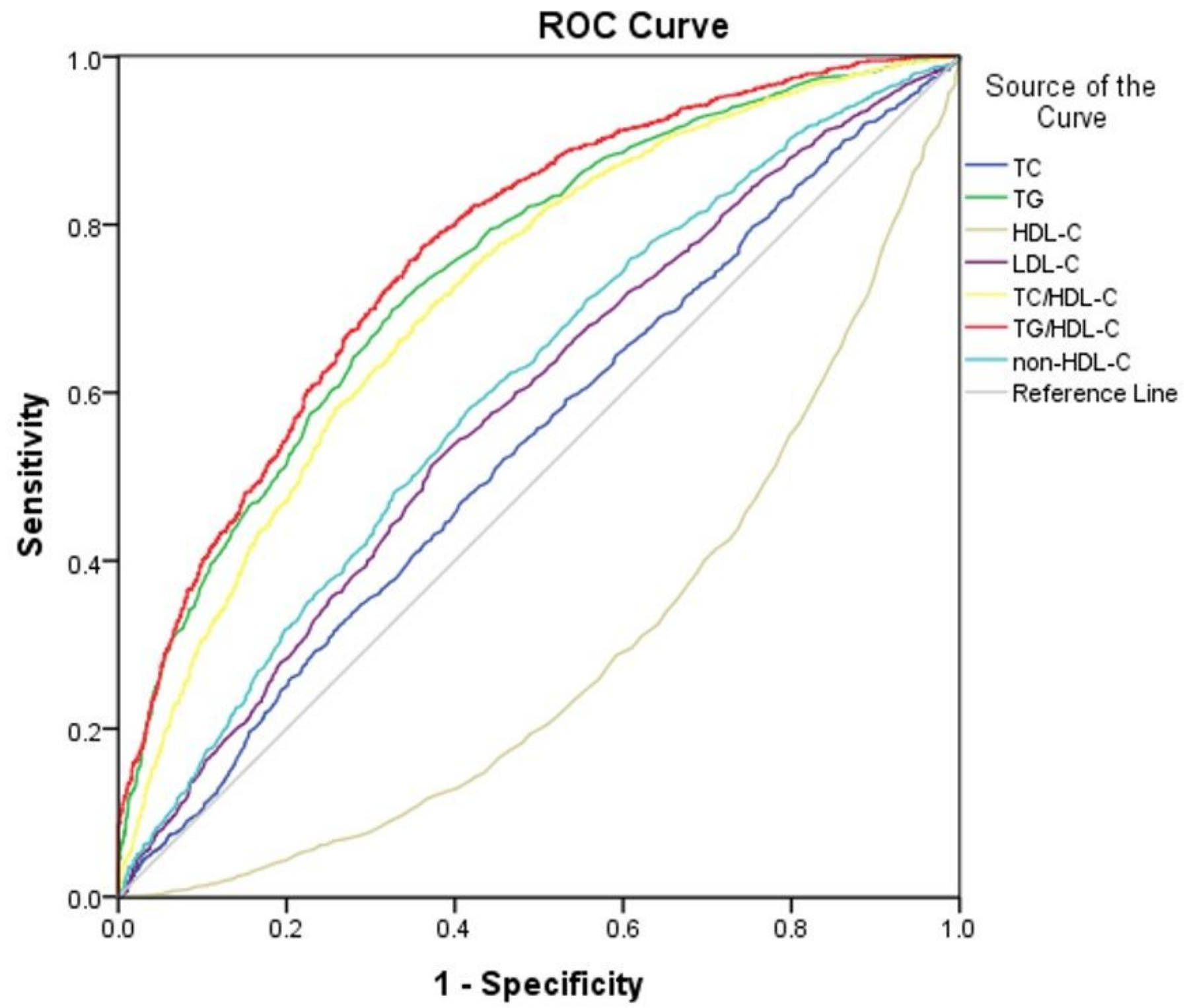

Figure 2

Area under the receiver operating characteristics curves (AUROCs) of parameters for NAFLD 


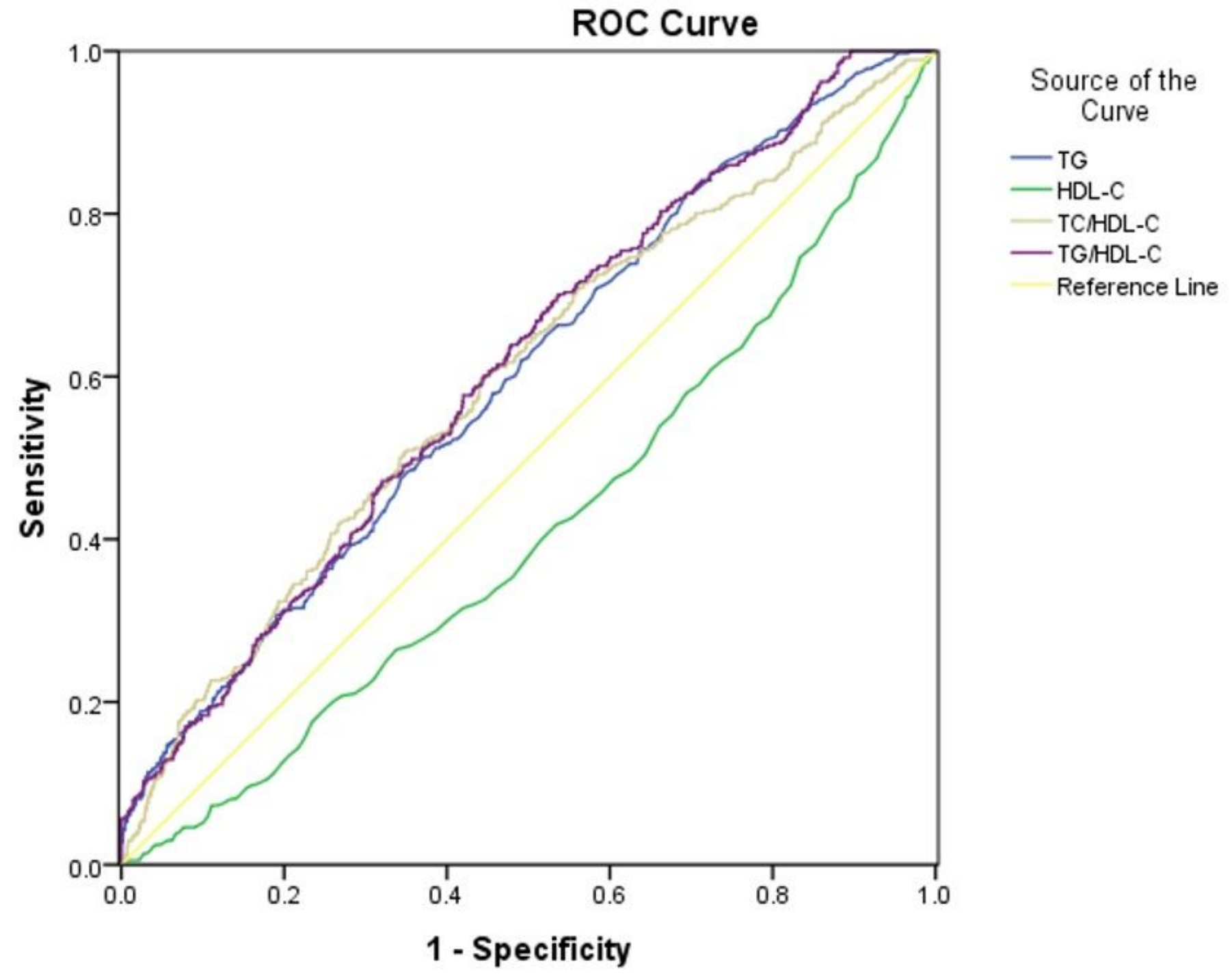

Figure 3

Area under the receiver operating characteristics curves (AUROCs) of parameters for liver fibrosis 